\title{
Nanogenerator-induced synaptic plasticity and metaplasticity of bio-realistic artificial synapses
}

\author{
Bo-Yun Kim ${ }^{1}$, Hyun-Gyu Hwang ${ }^{1}$, Jong-Un Woo' ${ }^{1}$, Woong-Hee Lee ${ }^{2}$, Tae-Ho Lee ${ }^{2}$, Chong-Yun Kang ${ }^{1,3}$ \\ and Sahn $\mathrm{Nahm}^{1,2}$
}

\begin{abstract}
A bio-realistic artificial synapse integrated with a nanogenerator (NG), which can be used in neuromorphic systems, is demonstrated for self-powered biomedical devices in this study. Biocompatible amorphous $\left(\mathrm{Na}_{0.5} \mathrm{~K}_{0.5}\right) \mathrm{NbO}_{3}(\mathrm{NKN})$ films are grown on TiN/polyimide substrates to synthesize NKN memristors for use as artificial synapses. Various synaptic functions are realized in NKN memristors, which are driven by a pulse generator and an NG. The synaptic plasticity of the NKN memristor results from the oxygen vacancy movements and the changes in the shape of the oxygen vacancy filaments. As a further step toward developing more bio-realistic artificial synapses, various types of metaplasticity and their mechanisms in the NKN memristors are investigated. Moreover, the metaplasticity of spike-timing-dependent plasticity (a key characteristic of biological synapses) is realized in the NKN memristor with a priming stimulus given by the NKN NG.
\end{abstract}

NPG Asia Materials (2017) 9, e381; doi:10.1038/am.2017.64; published online 26 May 2017

\section{INTRODUCTION}

Self-powered biomedical devices have been extensively investigated for their potential to eliminate battery use, increase device lifetime and allow for further miniaturization. ${ }^{1-3}$ In particular, biomedical devices driven by piezoelectric nanogenerators (NGs), which can convert wasted mechanical energy from the human body into electrical energy, have exhibited numerous advantages. ${ }^{3-5}$ Future advances in these self-powered biomedical devices will require higher functionality within a small volume. Thus, an energy-efficient computing system is important. However, traditional digital computing devices based on the von Neumann architecture are inappropriate for self-powered biomedical systems because of their high-power consumption and limits to potential miniaturization caused by their inefficient computing systems. ${ }^{6-8}$ Alternatively, neuromorphic computing systems (inspired by the extremely efficient human brain) have been explored as a promising technology. ${ }^{9,10}$ In particular, the development of artificial synapses that emulate the properties of biological synapses (as components of brain-like computing systems) is crucial for creating self-powered neuromorphic biomedical devices that can perform sophisticated functions and consume less power. ${ }^{8-10}$

Synaptic plasticity, which is the ability to continuously modulate synaptic weight, is a key property of biological synapses that must be mimicked in artificial synapses. ${ }^{11-13}$ This property includes the potentiation/depression of synaptic weight, spike-rate-dependent plasticity (SRDP) and spike-timing-dependent plasticity (STDP). ${ }^{11-13}$ In biological systems, synaptic plasticity can be modulated by inducing a priming stimulus before the main actions are taken to change synaptic weights, which is defined as metaplasticity. ${ }^{14-16}$ Specifically, metaplasticity changes the ability of biological synapses to generate synaptic plasticity and is considered to be a higher-order form of synaptic plasticity. ${ }^{14-16}$ Facilitating metaplasticity in artificial synapses to implement improved and bio-realistic synaptic functions is very challenging.

A memristor, which is a two-terminal resistor with a resistance that can be continuously adjusted, has been proposed for artificial synapses because its tunable analog resistance is similar to the adjustable synaptic weight of biological synapses. ${ }^{17-19}$ Various memory devices have been investigated for use in such memristors, including ferroelectric random access memory, ${ }^{20}$ resistive random access memory (ReRAM), ${ }^{21-24}$ and phase resistive random access memory. ${ }^{25}$ Among these devices, the ReRAM-based memristor is considered to be a promising candidate for artificial synapses because synaptic plasticity has been promoted well in ReRAM devices. ${ }^{17,21}$ Additionally, these devices consume lower amounts of power and have good compatibility with CMOS technology. ${ }^{17,26}$ Recently, metaplasticity has been reported in a $\mathrm{WO}_{3}$ system, which shows great potential as a bio-realistic artificial synapse, ${ }^{27}$ but further investigation of the various types of metaplasticity and their mechanisms needs to be performed.

Some recent studies have reported various devices integrated with NGs as a self-powered system. ${ }^{28-30}$ In our previous study, an amorphous $\left(\mathrm{Na}_{0.5} \mathrm{~K}_{0.5}\right) \mathrm{NbO}_{3}(\mathrm{NKN})$ thin film showed excellent ReRAM properties with good biocompatibility. A piezoelectric NG was also synthesized using the same amorphous NKN film. ${ }^{28}$

\footnotetext{
${ }^{1}$ Nano-Bio-Information-Technology, KU-KIST Graduate School of Converging Science and Technology, Korea University, Seoul, Republic of Korea; ${ }^{2}$ Department of Materials Science and Engineering Korea University, Seoul, Republic of Korea and ${ }^{3}$ Electronic Materials Research Center, Korea Institute of Science and Technology (KIST), Seoul, Republic of Korea

Correspondence: Professor S Nahm, Department of Materials Science and Engineering, Korea University, 145 Anam-ro, Seongbuk-Gu, Seoul 02841, Republic of Korea.

E-mail: snahm@korea.ac.kr

Received 10 January 2017; revised 17 February 2017; accepted 13 March 2017
} 
a

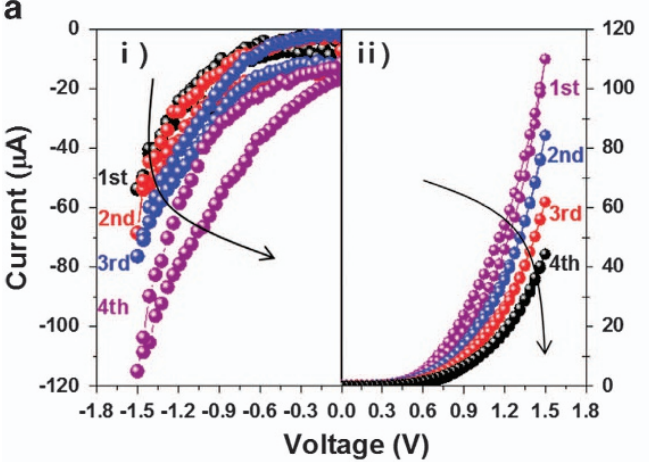

b

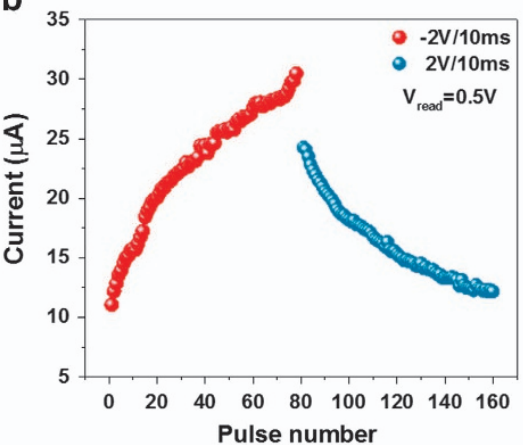

C

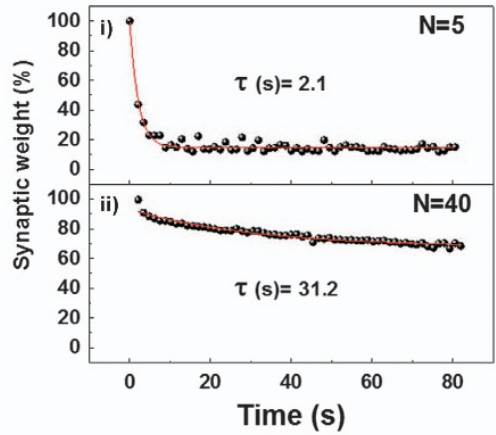

d i ) P-spike applications

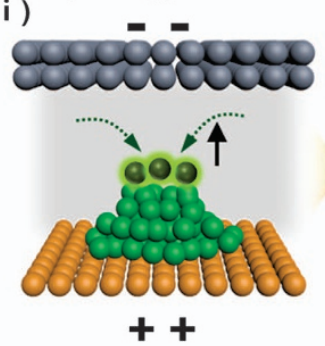
ii)

P-spike removal

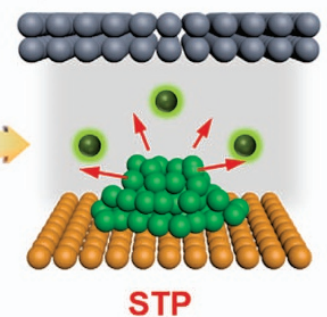

e

P-spike applications i )

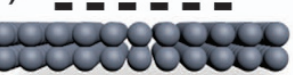

- Pt

TiN

O Oxygen vacancy

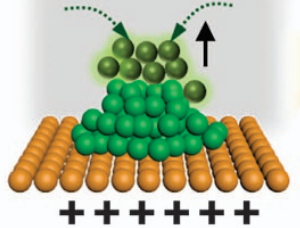

ii)

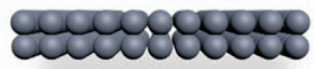

f

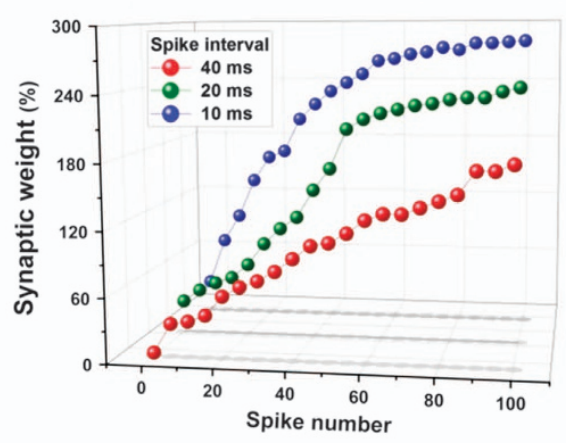

g

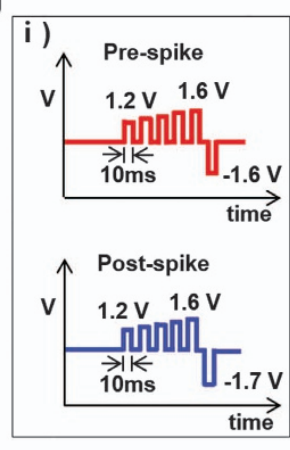

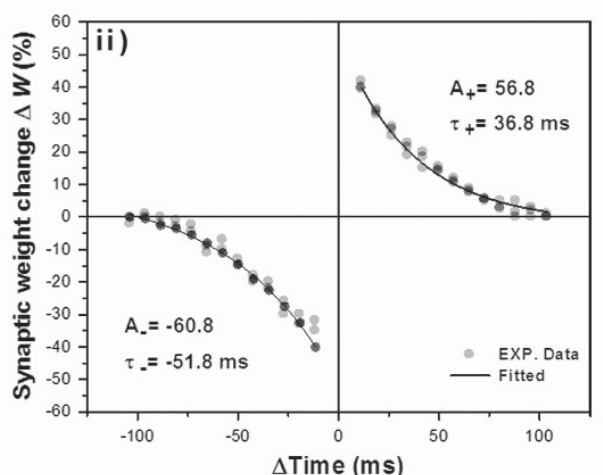

Figure 1 (a) $I-V$ characteristics of the NKN memristor measured under four consecutive negative (i) and positive (ii) voltages. (b) Current variations developed in the NKN memristor with applications of 80 P-spikes and 80 D-spikes. (c) Variations in synaptic weight with respect to the retention time after 5 (i) and 40 (ii) P-spikes. Pictorial representations for the movement of oxygen vacancies and the shape of the ruptured filaments for the (d) STP and (e) LTP processes occurring in the NKN memristor. (f) Variations in the synaptic weight of an NKN memristor with respect to the P-spike number and interval. (g) (i) Pre-spikes and post-spikes applied to the NKN memristor and (ii) variations in $\Delta W$ with respect to $\Delta t$.

Additionally, this NKN ReRAM, driven by an NKN NG, exhibited stable and desirable ReRAM properties. $^{28}$ In this study, we demonstrate an artificial synapse for self-powered biomedical devices in neuromorphic systems: a flexible NKN memristor driven by an NKN NG. Various types of synaptic plasticity were realized in the NKN memristor operated by the NKN NG. Finally, the metaplasticity of the memristor was investigated. The results of this study may provide new insight into the development of self-powered artificial synapses for neuromorphic bioelectronics.

\section{METHODS}

Device fabrication

A 120-nm-thick TiN bottom electrode was deposited on a polyimide (PI) substrate by electron-beam evaporation as the bottom electrode of the NKN memristors. Moreover, an 80 -nm-thick NKN film was grown on the TiN/polyimide substrates at $300^{\circ} \mathrm{C}$ using an RF magnetron sputtering system in a vacuum chamber under a mixture of $\mathrm{Ar}(80 \%)$ and $\mathrm{O}_{2}(20 \%)$ with a sputtering power of $80 \mathrm{~W}$. The working pressure was 10.0 mTorr. Pt (top) electrodes were deposited on the NKN films by DC sputtering (Emitech K550, Emitech Ltd., Ashford, Kent, UK) using a metal shadow mask to form a metal-insulator-metal structure. Therefore, the NKN memristor consists of Pt $(50 \mathrm{~nm}) / \mathrm{NKN}(80 \mathrm{~nm}) / \mathrm{TiN}(120 \mathrm{~nm}) /$ polyimide $(125 \mu \mathrm{m})$.

\section{Device measurement}

Electrical measurements were performed with a Keithley 4200 Semiconductor Characterization System and a Pulse Function Arbitrary Noise Generator (Agilent 81150A, Santa Clara, CA, USA). For measurement of synaptic plasticity and metaplasticity in the NKN memristor, all read pulse values were $0.5 \mathrm{~V} /$ $10 \mathrm{~ms}$. The temperature dependence of resistance was measured with a standard four-probe method using both a Physical Property Measurement System (Ever Cool II, Quantum Design, San Diego, CA, USA) and a custommade setup. 


\section{RESULTS AND DISCUSSION}

An NKN memristor grown on a polyimide substrate is appropriate for use as an artificial synapse because it shows a continuous change in resistance levels with good electrical and mechanical reliabilities (see Supplementary Sections S1-S3). Moreover, the low-resistance state (LRS) and high-resistance states of an NKN memristor can be explained by the formation and rupture of oxygen vacancy filaments, as shown in Supplementary Sections S4-S8. To investigate the synaptic transmission characteristics of the NKN memristor, the $I-V$ characteristics of the memristor were measured under four consecutive negative and positive voltages, as shown in Figures $1 \mathrm{a}-\mathrm{i}$ and a-ii. The conductance was increased when consecutive negative sweeps were applied to the memristor, and it decreased under positive voltage sweeps. These results show that the repetitive application of a DC bias can control the conductance of the NKN memristor. The conductance modulation of the NKN memristor corresponds to the nonlinear transmission behavior observed in biological synapses. ${ }^{18,31}$ Furthermore, 80 negative and positive pulses were applied to the NKN memristor as potentiation spikes (P-spikes) and depression spikes (D-spikes), respectively, to implement the potentiation and depression of synaptic weights in the NKN memristor (see Figure 1b). The conductance of the NKN memristor increased (or decreased) as the number of P-spikes (or D-spikes) increased. The modulation of the NKN memristor conductance with the number of applied $\mathrm{P}$ - and D-spikes mimics the tunable synaptic weight of biological synapses. ${ }^{11-13,22}$

The synaptic short-term plasticity (STP) and long-term plasticity $(\mathrm{LTP})^{32,33}$ of the NKN memristor have also been investigated. Figure $1 c-i$ and $c$-ii show the variation in synaptic weights with respect to the retention time after the memristor was subjected to five and 40 P-spikes, respectively. The synaptic weight of the NKN memristor decreased rapidly after the removal of the five P-spikes, and a very small stable synaptic weight remained, as shown in Figure 1c-i. After the application of 40 P-spikes, however, the decrease in synaptic weight was not significant, and a considerably increased stable synaptic weight was observed, as shown in Figure 1c-ii. Therefore, LTP can be induced in the NKN memristor by increasing the number of P-spikes. The relaxation curve for the synaptic weight of the NKN memristor is very similar to the memory-forgetting curve of the human brain. ${ }^{19,22,34}$ The behavior of human memory is usually expressed by a simple stretched-exponential function, as shown in Equation (1). ${ }^{34}$ This equation was used to investigate the synaptic weight relaxation behavior of the NKN memristor.

$$
W(t)=W_{\mathrm{e}}+A \exp (-t / \tau)
$$

$W(t)$ and $W_{\mathrm{e}}$ are the synaptic weights of the NKN memristor at time $t$ and the synaptic weight of the stable state, respectively. $A$ is a constant and $\tau$ is the relaxation time constant, which is an important factor for evaluating the decay rate of the synaptic weight. Relaxation curves for the synaptic weight of the NKN memristor were fitted using Equation (1) (see Figure $1 \mathrm{c}-\mathrm{i}$ and c-ii), and $\tau$ and $W_{\mathrm{e}}$ were obtained. A small $\tau$ value of $2.1 \mathrm{~s}$ with a small stable synaptic weight of $15 \%$ and an increased $\tau$ value of $31.2 \mathrm{~s}$ with a large stable synaptic weight of $68 \%$ were obtained from the estimated relaxation curves after 5 and $40 \mathrm{P}$-spike stimulations, respectively. These results indicate that increasing the number of P-spikes increased both values of $\tau$ (i.e., a slower decay rate) and $W_{\mathrm{e}}$. Other relaxation behaviors of the NKN memristor, which were formed after the application of different numbers of P-spikes, are also shown in Supplementary Figures S10a-c in Section 9 of the Supplementary Information.
Figures $1 \mathrm{~d}$ and e show schematic diagrams of the filaments formed in the NKN memristor for the STP and LTP processes, respectively. When five P-spikes were applied to the NKN memristor that was in a long-term state, a few of the oxygen vacancies moved into the pre-existing filament as a result of the electrical energy supplied by the P-spikes (see Figure 1d-i). This change resulted in an instantaneous increase in current. However, these oxygen vacancies immediately diffused back after the P-spikes were stopped due to the concentration gradient of oxygen vacancies in the NKN film, as shown in Figure 1d-ii. The current then significantly decreased, and the STP can be explained by the behavior of these oxygen vacancies. In contrast, when 40 spikes were applied to the NKN memristor, many oxygen vacancies moved into the filament (see Figure 1e-i) and remained there because sufficient electrical energy was continuously supplied to the oxygen vacancies during the application of the 40 P-spikes. Finally, the added oxygen vacancies led to filament growth, which resulted in increases in filament length even after the P-spikes were stopped, as shown in Figure 1e-ii. Therefore, the current permanently increased, and this condition corresponds to a new stable state. This new long-term state is shown in Figure 1e-ii, and it can be explained by a decrease in the gap sizes between the oxygen vacancy filament and electrode.

Figure 1f shows the variation in synaptic weight with respect to the P-spike number and interval. The synaptic weight increased with an increasing number of P-spikes. Additionally, the NKN memristor exhibited a larger increase in synaptic weight when P-spikes with a shorter interval were applied to the NKN memristor. The largest synaptic weight was observed when P-spikes with a 10-ms interval were applied to the NKN memristor. This result corresponds to the SRDP characteristic of a biological synapse, which demonstrates that frequent stimulations lead to larger changes in the synaptic weight. ${ }^{33}$ The SRDP exhibited by the NKN memristor can be explained as follows. The oxygen vacancy migration occurs as a result of the applied P-spikes. After the removal of the P-spikes, the oxygen vacancies then move continuously due to the inertia effect. When the next P-spike is applied to the NKN memristor (before oxygen vacancy movement has stopped), the movement of the oxygen vacancies can be accelerated. Therefore, P-spikes with a short interval increased the movement of oxygen vacancies, which resulted in a larger increase in synaptic weight. Similar results were observed in the $\mathrm{InGaZnO}, \mathrm{WO}_{x}$ and $\mathrm{HfO}_{x}$ memristors. ${ }^{22,31,34}$

To realize STDP in the NKN memristor, pre- and post-spikes (see Figure 1g-i) were applied to the Pt (top) and TiN (bottom) electrodes, respectively. The actual net spike (the difference between the pre- and post-spikes) applied to the NKN memristor is determined by $\Delta t$. Supplementary Figures S11a-c in Section 10 of the Supplementary Information show several examples of net spikes applied to the NKN memristor with different $\Delta t$ values. Figure 1g-ii shows the relative changes in synaptic weight $(\Delta W)$ with respect to $\Delta t$. Potentiation and depression of the synaptic weight were observed at $\Delta t>0$ and $\Delta t<0$, respectively. Generally, STDP in a biological synapse has been expressed using the following equation: ${ }^{22}$

$$
\Delta W= \begin{cases}A_{+} \mathrm{e}^{-|\Delta t| \tau_{+}}+\Delta W_{0+}, & \Delta t>0 \\ A_{-} \mathrm{e}^{-|\Delta t| \tau_{-}}+\Delta W_{0-}, & \Delta t<0\end{cases}
$$

$A_{+} / A_{-}$and $\tau_{+} / \tau_{-}$are scaling factors and time constants, respectively. The experimental results shown in Figure 1g-ii were estimated using Equation (2). The $A_{+} / A_{-}$and $\tau_{+} / \tau_{-}$values obtained were $56.8 /-60.8$ and $36.8 \mathrm{~ms} /-51.8 \mathrm{~ms}$, respectively. The STDP curve of the NKN memristor was in good agreement with the biological synaptic characteristics reported by $\mathrm{Bi}$ and Poo. ${ }^{11,35,36}$ 


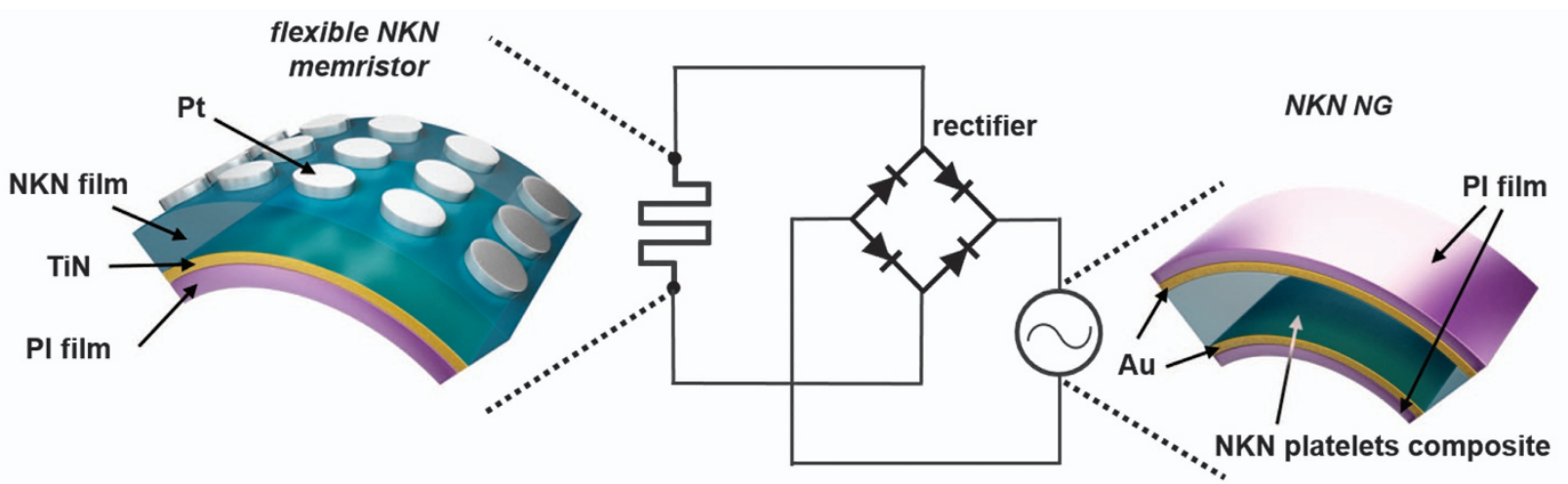

b

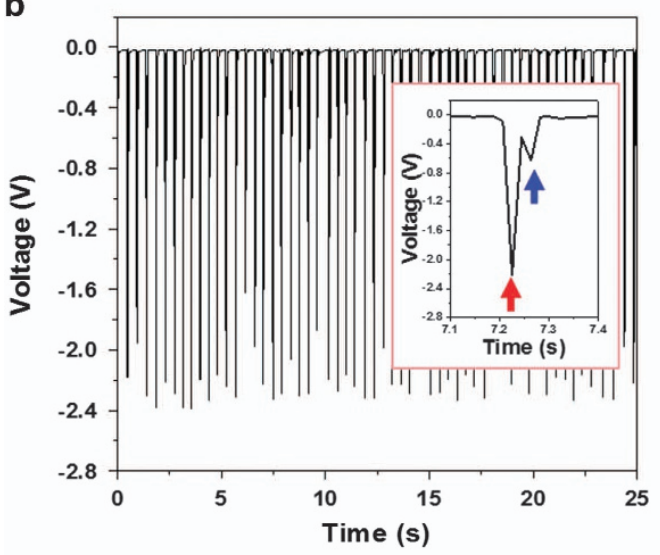

d

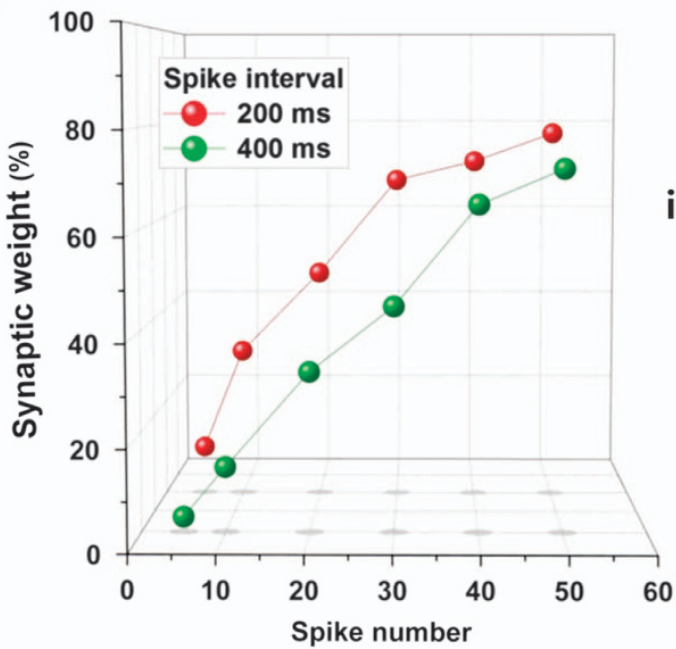

C

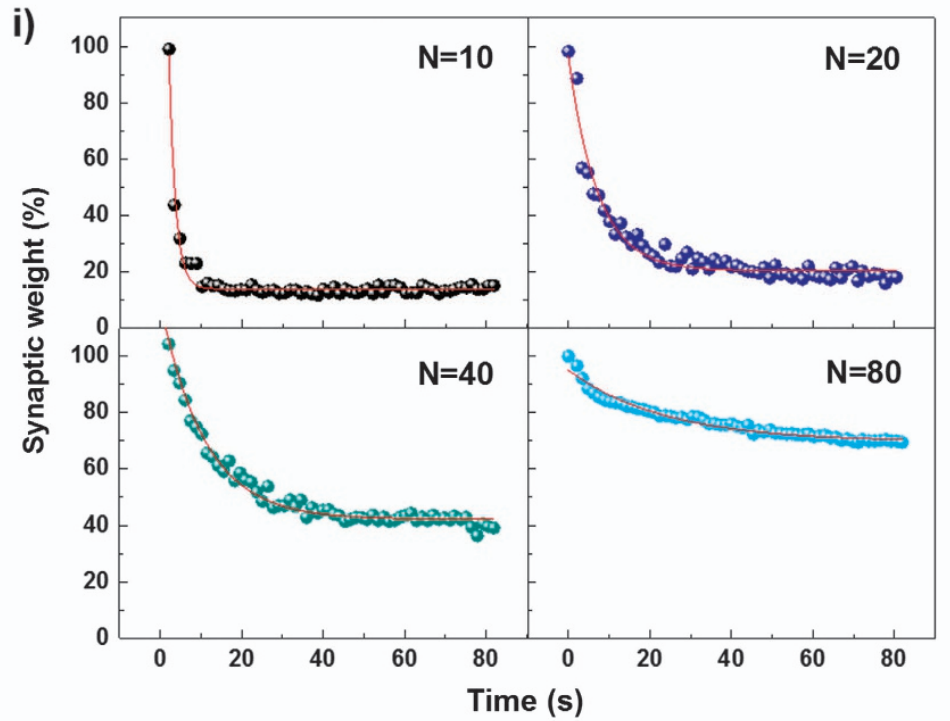

ii) 30

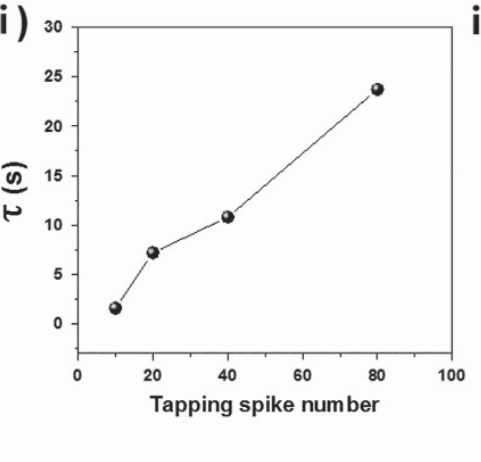

iii)

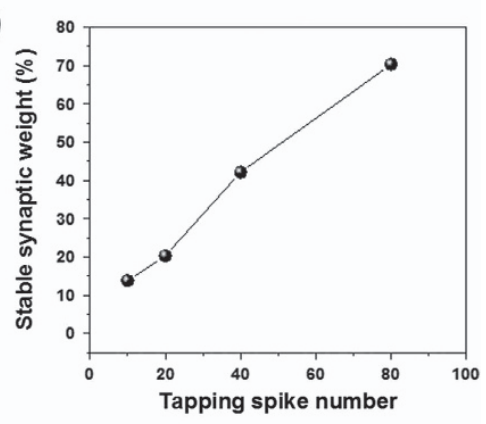

Figure 2 (a) A bridge rectifier circuit diagram of the NKN memristor and NKN NG. (b) Open circuit output voltage of the NKN NG after rectification. (c) (i) Variation of synaptic weight with respect to the retention time at different P-spike numbers, and (ii) relaxation time and (iii) stable synaptic weight with respect to the P-spike number. (d) Variations of the synaptic weight with respect to the P-spike number and interval.

The NKN memristor was integrated with an NKN NG, as shown in Figure $2 \mathrm{a}$, to explore applications for self-powered artificial synapses. The synaptic plasticity of the NKN memristor driven by the NKN NG was investigated. The piezoelectric NG was fabricated using NKN platelets and was operated manually (by finger tapping). ${ }^{37}$ Figure $2 \mathrm{~b}$ shows the output voltages generated by the NKN NG after negative rectification. The red and blue arrows in the inset of Figure $2 \mathrm{~b}$ indicate the output voltages generated by pressing and releasing the NG, respectively. When the NG was pressed, a large average output voltage $(>-2.0 \mathrm{~V})$ was obtained with an average pulse width of approximately $11 \mathrm{~ms}$ at $-2.0 \mathrm{~V}$. The output voltages and output currents generated by the NKN memristors are shown in Supplementary Figures S12a and b, respectively, in Section 11 of the Supplementary Information. Since the effective voltage for the potentiation of the NKN memristor was approximately $-2 \mathrm{~V} / 10 \mathrm{~ms}$, the output voltage obtained from the NKN NG can be used as the P-spike. The output voltage generated by releasing the NG was too small to change the synaptic weights and was thus neglected.

Figure $2 \mathrm{c}-\mathrm{i}$ shows variations in synaptic weight with respect to the retention time for an NKN memristor stimulated with various 
a
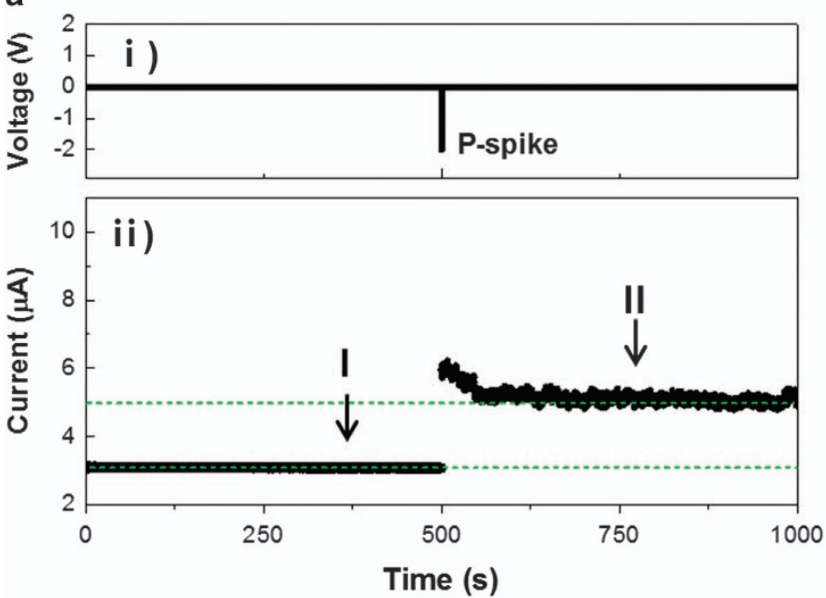

C

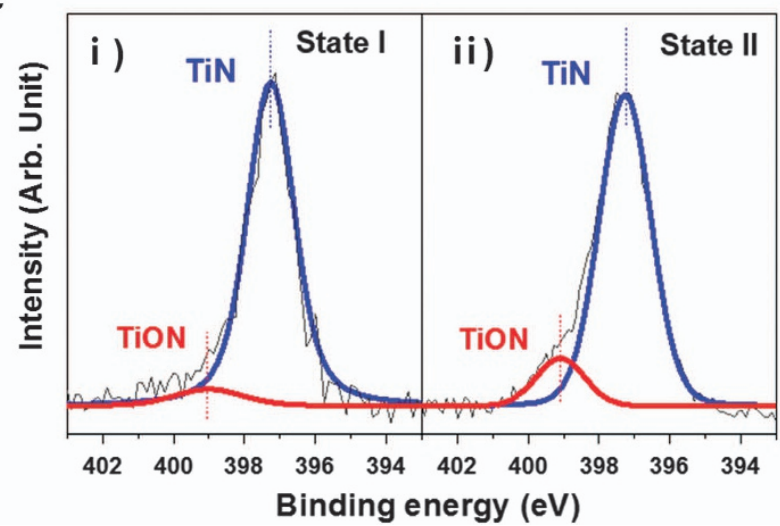

b
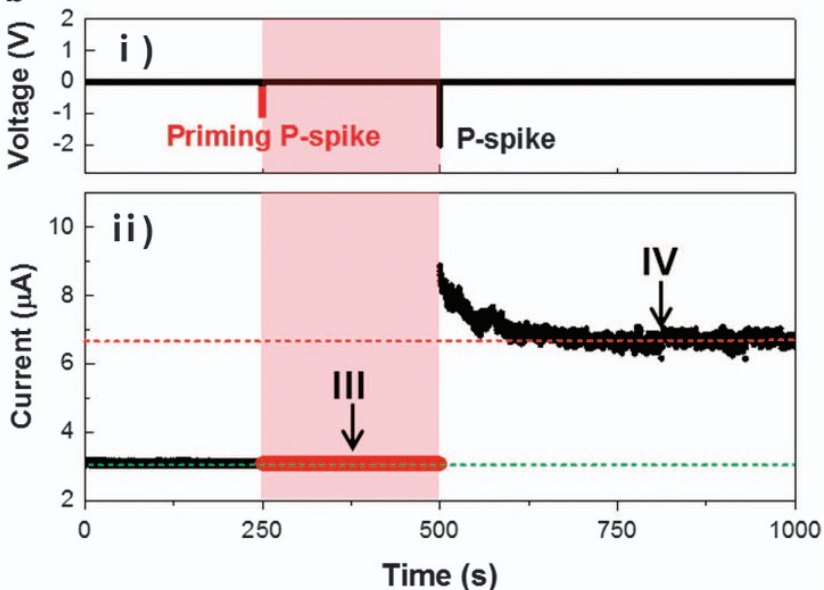

d

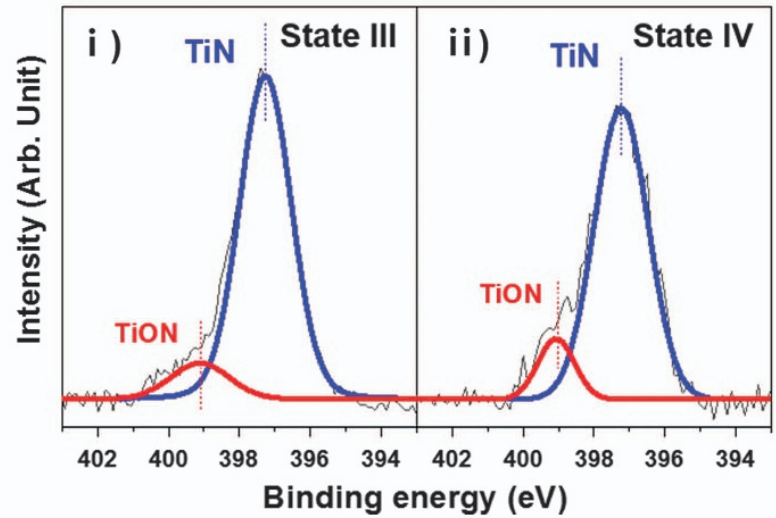

Figure 3 (a) (i) P-spike applied to the NKN memristor and (ii) current of the NKN memristor in state I and state II. (b) (i) Priming P-spike and P-spike applied to the NKN memristor and (ii) current of the NKN memristor in state I, state III and state IV. (c, d) X-ray photoelectron spectroscopy spectra taken from the NKN film near the NKN/TiN interface in various states: (c-i) state I, (c-ii) state II, (d-i) state III and (d-ii) state IV.

numbers of NG-produced P-spikes. Generally, the synaptic weight increased as the number of P-spikes increased. These synaptic weight curves were also fitted using Equation (1) to obtain both $\tau$ and $W_{\mathrm{e}}$. The $\tau$ value increased with an increase in the number of P-spikes (see Figure 2c-ii), which indicated a decrease in the decay rate of the synaptic weight. $W_{\mathrm{e}}$ also increased as the number of P-spikes increased, as shown in Figure $2 \mathrm{c}$-iii. These results are very similar to the results for the NKN memristor operated by a pulse generator, which shows that synaptic plasticity (including STP and LTP transitions) can be reproduced in an NKN memristor operated by an NKN NG. Compared to the NKN memristor operated by a pulse generator, the smaller $\tau$ and lower $W_{\mathrm{e}}$ of the memristor driven by the NG can be explained by the larger P-spike interval produced from manual motion (see Supplementary Figures S10b and $c$ in Section 9 of the Supplementary Information).

SRDP was also investigated in the NKN memristor operated by the NG. Figure $2 \mathrm{~d}$ shows variations in synaptic weight as a function of the P-spike number for the NKN memristor operated by the NG with spike intervals of 200 and $400 \mathrm{~ms}$. The synaptic weight increased as the number of P-spikes increased. Smaller P-spike intervals led to larger increases in synaptic weight. This behavior is consistent with the outcomes observed with the NKN memristor operated by a pulse generator (see Figure 1f).

Metaplasticity in a biological synapse is defined as a higher-order form of synaptic plasticity, which entails changes in the ability of the synapse to generate synaptic plasticity by introducing priming stimuli before the application of a learning action through the main spikes. ${ }^{14-16}$ In this study, we also investigated the metaplasticity of the NKN memristor. The metaplasticity of the NKN memristor was measured by the commercial equipment. The NKN memristor in the high-resistance state (state I) has a current of $3.0 \mu \mathrm{A}$, as shown in Figure 3a-ii. When this device was stimulated by a P-spike of $-2 \mathrm{~V} / 100 \mathrm{~ms}$ (see Figure $3 \mathrm{a}-\mathrm{i}$ ), the NKN memristor was potentiated (state II). It then showed an increased current of $5.0 \mu \mathrm{A}$ (see Figure 3a-ii). This increase constitutes conventional long-term potentiation behavior. On the other hand, when a priming P-spike of $-1 \mathrm{~V} / 10 \mathrm{~ms}$ was applied to the NKN memristor before a standard P-spike (see Figure 3b-i), the current of the NKN memristor was not changed (state III), as shown in Figure 3b-ii. However, when the $\mathrm{P}$-spike was applied to the NKN memristor after the priming P-spike, the current of the NKN memristor increased to $6.7 \mu \mathrm{A}$ (state IV), which is larger than the current produced by conventional synaptic potentiation $(5.0 \mu \mathrm{A})$ (see Figure 3b-ii). Therefore, although the priming P-spike did not increase the current of the NKN memristor, it enhanced its ability to produce long-term potentiation, which resulted in an increased current of $6.7 \mu \mathrm{A}$ when the P-spike was applied to the NKN memristor. This result suggests that metaplasticity with respect to long-term potentiation can be implemented in the NKN memristor. 
a
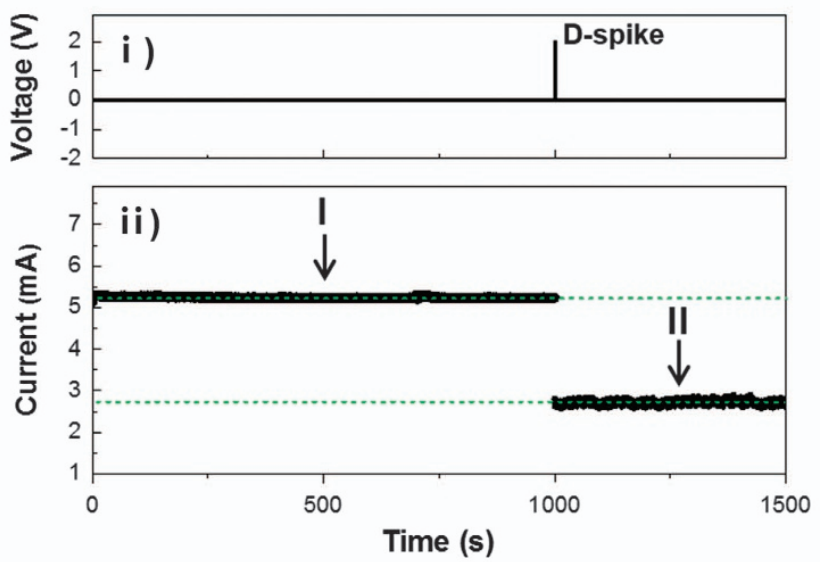

C

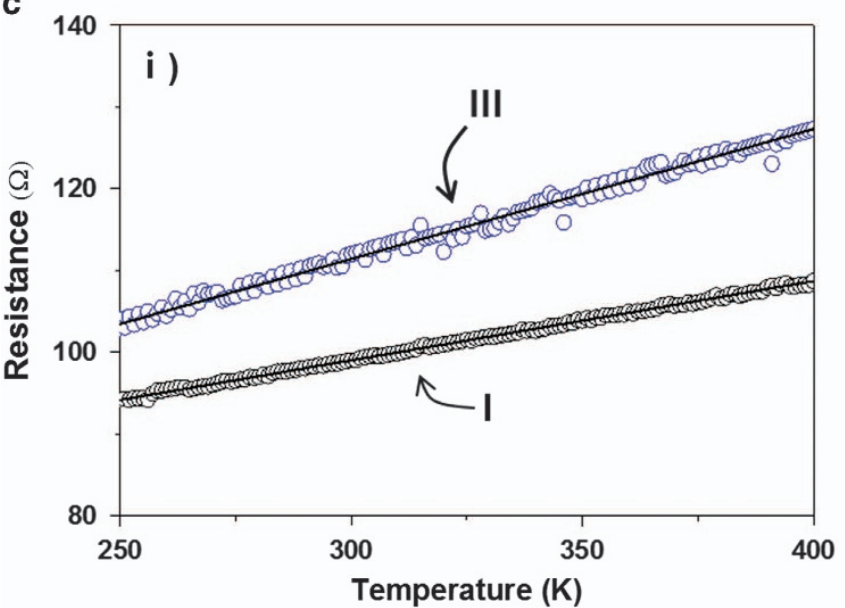

b
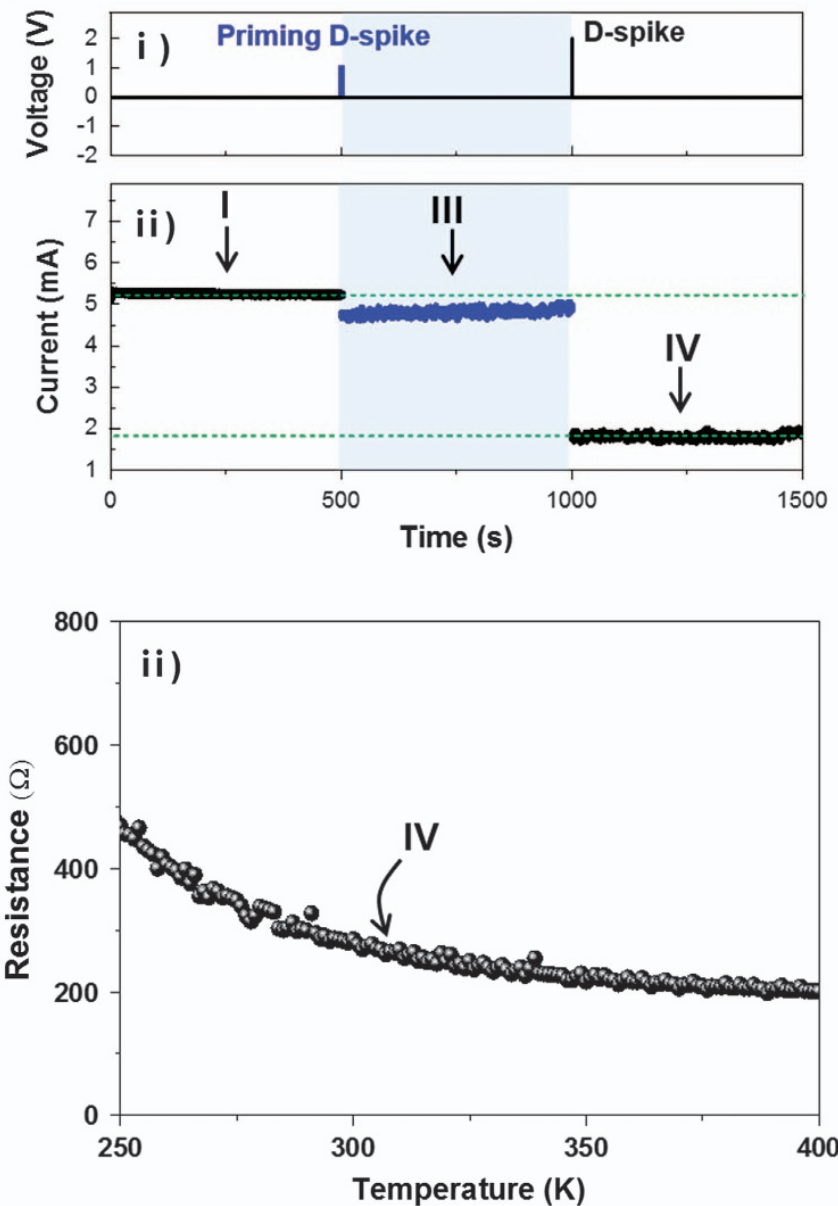

Figure 4 (a) (i) D-spike applied to the NKN memristor and (ii) the current of the NKN memristor in state I and state II. (b) (i) Priming D-spike and D-spike applied to the NKN memristor and (ii) current of the NKN memristor in state I, state III and state IV. (c) Temperature dependence of the resistance of the NKN memristor in (i) states I and III and (ii) state IV.

To investigate the metaplasticity mechanism for long-term potentiation in the NKN memristor, an X-ray photoelectron spectroscopy analysis was conducted at the NKN/TiN interface of an NKN memristor in various states (I, II, III and IV states). Figure 3c-i and c-ii show the normalized N1s spectra taken from the TiN electrode close to the NKN/TiN interface of an NKN memristor in states I and II, respectively. These spectra have two peaks at $396.79 \mathrm{eV}(\mathrm{TiN})$ and $398.6 \mathrm{eV}(\mathrm{TiON}){ }^{28,38}$ In state I, a strong TiN peak and a very weak TiON peak were observed, which indicates that there are few oxygen ions near the NKN/TiN interface. However, the intensity of the TiON peak increased after application of the P-spike (state II), as shown in Figure 3c-ii. Therefore, oxygen ions in the NKN film are considered to have diffused into the NKN/TiN interface as a result of the application of the P-spike, which forms the $\mathrm{TiO}_{x} \mathrm{~N}_{y}$ phase. ${ }^{28,38}$ Moreover, the oxygen vacancies created in the NKN film increased the length of the filament (or decreased the gap between the Pt and the filament) and increased the current $(5 \mu \mathrm{A})$ in state II of the NKN memristor. For state III of the NKN memristor (which develops after the priming P-spike), the intensity of the TiON peak slightly increased, as shown in Figure $3 \mathrm{~d}-\mathrm{i}$, which indicates that a small amount of the oxygen ions diffused into the TiN/NKN interface and increased TiON bonding. Therefore, it can be suggested that a small number of oxygen vacancies were formed in the NKN films. These oxygen vacancies did not increase the length of the filament, however, because the current of state III is the same as that of state I. However, when the P-spike was applied after the priming spike (state IV), the intensity of the TiON peak increased considerably, and the TiN peak decreased slightly, as shown in Figure 3d-ii. The result indicates that a large number of oxygen ions moved into the TiN/NKN interface because of both the priming $\mathrm{P}$-spike and the P-spike, thus forming the TiON phase. Moreover, the oxygen vacancies produced in the NKN film actually increased the length of the filaments when the P-spike was applied, resulting in a large current $(6.7 \mu \mathrm{A})$ in the $\mathrm{NKN}$ memristor in state IV. Both the priming P-spike and the P-spike produced oxygen vacancies in stage IV, and the number of oxygen vacancies in stage IV was larger than that in stage II as a result. Thus, the length of the filament in stage IV could be larger than that in state II, which resulted in a higher current in stage IV. The priming P-spike contributed to an increase in the current of the NKN memristor after P-spike stimulation, although it did not immediately induce a current change in the NKN memristor. This result suggests that the priming P-spike improved the ability of the NKN memristor for long-term potentiation, which demonstrates metaplasticity for long-term potentiation.

Metaplasticity in relation to long-term depression of the $\mathrm{NKN}$ memristor was also investigated. As an example, the NKN memristor in the LRS (state I) exhibited a current of $5.3 \mathrm{~mA}$, as shown in Figure 4a-ii. When a D-spike of $2 \mathrm{~V} / 100 \mathrm{~ms}$ was applied to the NKN 
a

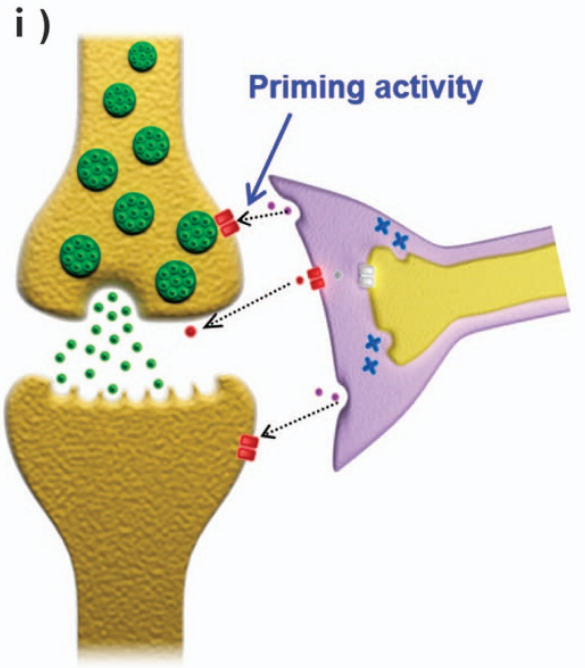

ii)

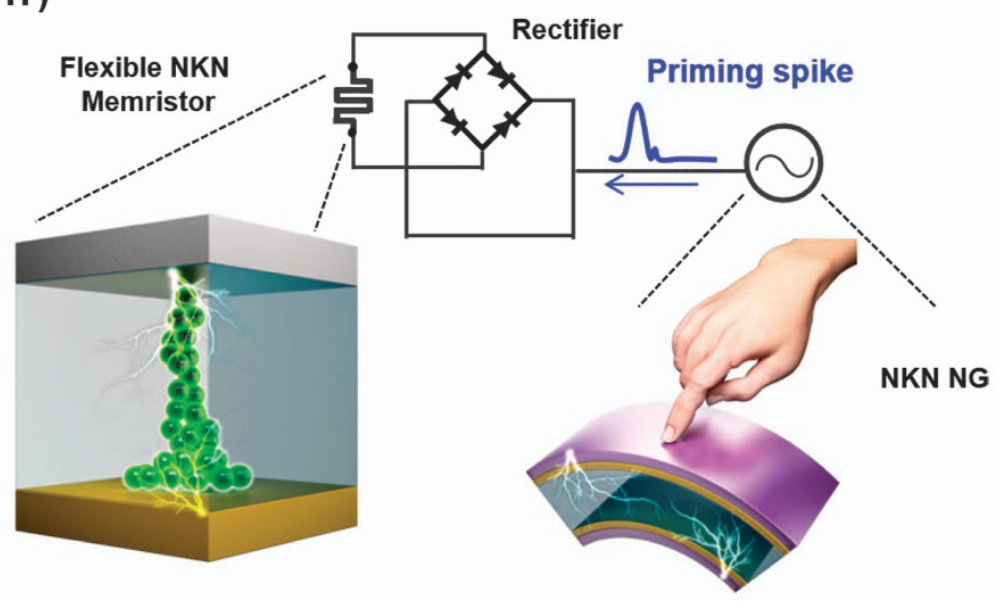

b

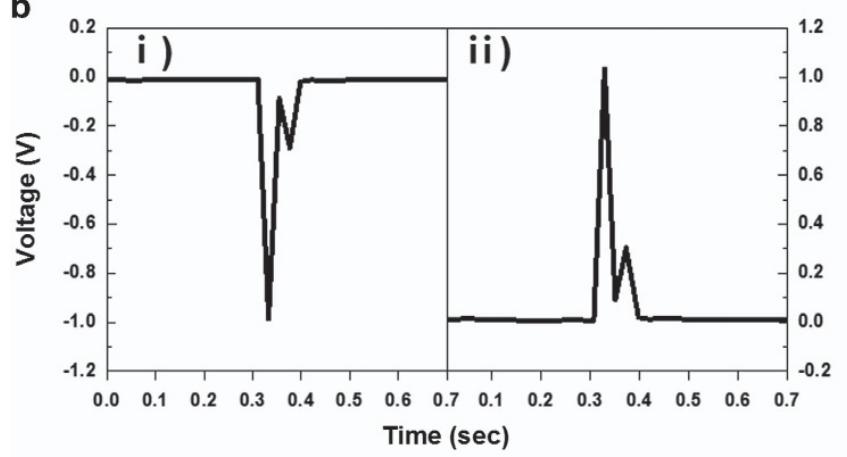

d

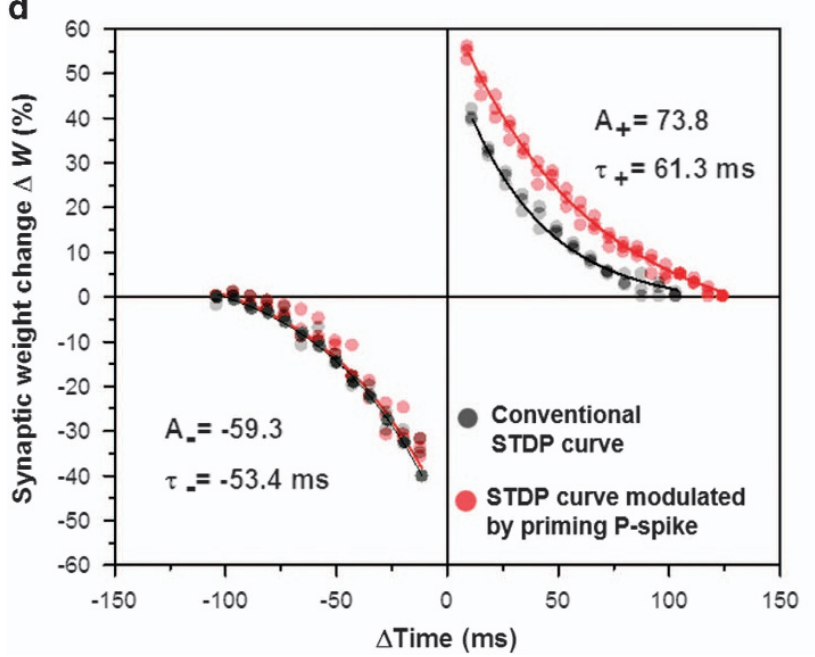

C
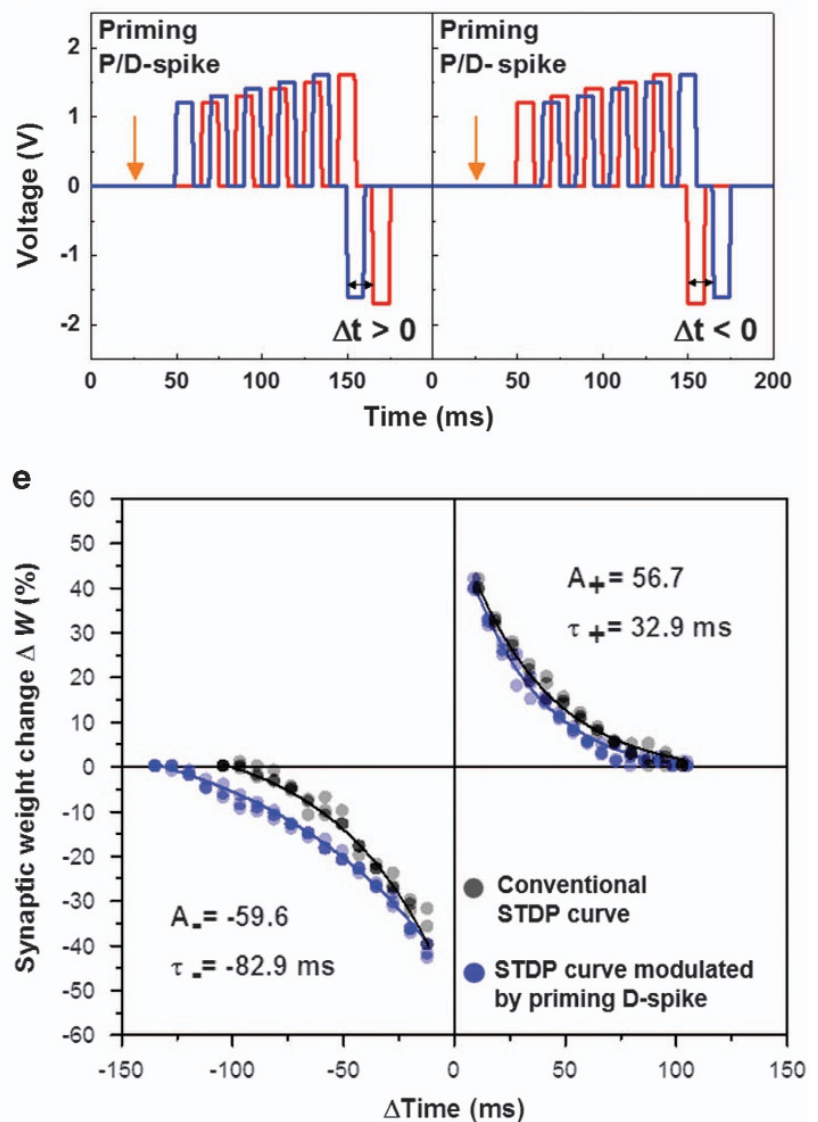

Figure 5 (a) Pictorial representations (i) of a biological synapse operated by astrocytes for metaplasticity and (ii) an NKN memristor operated by the NKN NG for metaplasticity. (b) Open circuit output voltage generated by the NKN NG after rectification for the (i) priming P-spike and (ii) priming D-spike. (c) Schematic diagram for a pulse train consisting of a priming spike, a pre-spike and a post-spike for implementing metaplasticity of STDP in the NKN memristor. Variations in $\Delta W$ with respect to $\Delta t$ when the (d) priming P-spike and P-spike and (e) priming D-spike and D-spike were applied to the NKN memristor using the NKN NG.

memristor (see Figure 4a-i), the current was reduced to $2.8 \mathrm{~mA}$ (state II), which corresponds to conventional long-term depression. On the other hand, when a priming D-spike of $1 \mathrm{~V} / 10 \mathrm{~ms}$ was applied to the NKN memristor (see Figure 4b-i), the current was slightly reduced from 5.3 to $4.8 \mathrm{~mA}$ (stage III). Furthermore, when a D-spike was applied to the NKN memristor after a priming D-spike (stage IV), the current was reduced to $1.8 \mathrm{~mA}$, as shown in Figure $4 \mathrm{~b}$-ii. It is interesting to note that the current of the LRS decreased slightly after the application of the priming D-spike. If the decrease of the current from the priming $\mathrm{D}$-spike (which is the current difference between 
state I and state III) was the same as the current difference between state II and state IV, the metaplasticity of long-term depression in the NKN memristor could not be realized. However, since the current difference between state II and state IV $(1.0 \mathrm{~mA})$ is larger than the decrease in current due to the priming D-spike $(0.5 \mathrm{~mA})$, the NKN memristor is considered to exhibit metaplasticity of long-term depression as well.

To identify the metaplasticity mechanism for long-term depression, variations in resistance with respect to temperature were measured for the NKN memristor in various states (I, III and IV), as shown in Figure 4c-i and c-ii. The resistances of the NKN memristor in states I and III increased with increasing temperature, as shown in Figure 4c-i, which indicates that the NKN memristor in these states exhibits a metallic-conducting property. On the other hand, the resistance of the NKN memristor in state IV decreased with increasing temperature (see Figure 4c-ii), which implies that the NKN memristor in this state exhibits an insulating property. The NKN memristor in state I was in the LRS, and the conducting filament, therefore, behaved as a current path. Moreover, because the NKN memristor in state III also exhibits metallic conduction characteristics, the filaments still behaved as a current path in this state. In general, the resistance of the memristor containing conducting filaments is determined by the diameter or thickness of the filament. ${ }^{39}$ The priming D-spike reduced the thickness of the conducting filaments without rupturing those filaments, which resulted in only a slight decrease in the current of the NKN memristor. On the other hand, when a D-spike was applied after a priming D-spike (state IV), the NKN memristor exhibited insulating properties, which indicates that the conducting filament had ruptured. Since the filament became thinner after the application of a priming D-spike, it could be easily ruptured by the application of a D-spike. Therefore, the ruptured filaments in state IV are considered to be thinner and smaller than the ruptured filament in state II. As a result, a smaller current was generated in state IV compared to state II, as shown in Figure $4 \mathrm{~b}$-ii. Schematic diagrams of the filament shape for each state are shown in Supplementary Figures S13a and b in Section 12 of the Supplementary Information. Based on the above results, it can be suggested that through a decrease in filament thickness, the priming D-spike improved the ability for long-term depression without changing the state of the NKN memristor from the LRS to the high-resistance state.

In the metaplasticity of biological synapses, an astrocyte produces priming activity in the neuronal synapse and modulates the synaptic plasticity of the synapse, as shown in Figure $5 \mathrm{a}-\mathrm{i} .{ }^{19-21}$ In this study, the NKN NG was used to apply priming spikes to the NKN memristor for the implementation of metaplasticity, as shown in Figure 5a-ii. In particular, the effect of metaplasticity on STDP was investigated for the NKN memristor using priming spikes produced from the NKN NG. Figure $5 \mathrm{~b}-\mathrm{i}$ and $\mathrm{b}$-ii show priming $\mathrm{P}$ - and $\mathrm{D}$-spikes with a maximum amplitude of $\pm 1.0 \mathrm{~V}$. Pulse trains consisting of priming spikes, pre-spikes and post-spikes were developed to induce metaplasticity for the STDP in the NKN memristor, as shown in Figure 5c. The pre- and post-spikes were identical to the spikes used to study conventional STDP in the NKN memristor (see Figure 1g-i). Figure 5d shows both conventional STDP curves (black circles) and STDP curves (red circles), which show the effects of priming P-spikes for metaplasticity. The priming P-spike increased $\Delta W$ and the range of $\Delta t$ (time window) at $\Delta t>0$, but variations in $\Delta W$ and the time window at $\Delta t<0$ were negligible. STDP curves showing the effects of priming P-spikes for metaplasticity were estimated using Equation (2). We obtained $A_{+} / A_{-}=73.8 /-59.3$ and $\tau_{+} / \tau_{-}=61.3 \mathrm{~ms} /-53.4 \mathrm{~ms}$. The $A_{+}$and $\tau_{+}$values increased from 58 to 73.8 and from $36.8 \mathrm{~ms}$ to
$61.3 \mathrm{~ms}$, respectively, by applying the priming P-spike, but the values of $A_{-}$and $\tau_{-}$were almost the same as the values for the conventional STDP curve. In the STDP curve, the larger values of $A_{+}$and $\tau_{+}$involve increases in $\Delta W$ and the time window at $\Delta t>0$, respectively. Moreover, since the threshold for potentiation decreases with an increasing STDP time window, increases in $\tau_{+}$reduced the potentiation threshold at $\Delta t>0$. The above results indicate that the priming $\mathrm{P}$-spike induces strong

and sensitive potentiation in STDP compared to potentiation in conventional STDP, which indicates that metaplasticity is exhibited in the potentiation of the STDP for the NKN memristor with the NKN NG.

Conventional STDP curves (black circles) and STDP curves showing the effects of metaplasticity from a priming D-spike (blue circles) are also shown in Figure 5e. A priming D-spike rarely changes potentiation but modifies depression in STDP. The STDP curves resulting from the use of a priming $\mathrm{D}$-spike were also estimated using Equation (2), and we obtained results of $A_{+} / A_{-}=56.7 /-59.6$ and $\tau_{+} / \tau_{-}=32.9 \mathrm{~ms} /-82.9 \mathrm{~ms}$. The $A_{+}$and $\tau_{+}$values did not change with the application of a priming D-spike. However, the absolute value

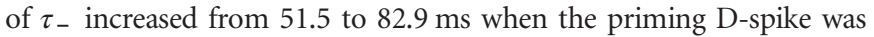
applied. Increasing the absolute value of $\tau_{-}$increases the time window for depression and eventually reduces the STDP depression threshold. As a result, the priming D-spike increases the sensitivity of depression in the STDP, which indicates that metaplasticity in the depression is also exhibited in the STDP of the NKN memristor with the NKN NG.

\section{CONCLUSION}

In summary, a flexible NKN memristor integrated with an NKN NG was demonstrated as a bio-realistic artificial synapse for self-powered biomedical device applications. Various forms of synaptic plasticity, including STP/LTP transition and SRDP, were implemented in the NKN memristor by controlling the number and frequency of P-spikes generated by a pulse generator as well as the NKN NG. Furthermore, metaplasticity for both long-term potentiation and depression was observed in the NKN memristor. This metaplasticity likely arose from variations in the shape of the filaments. Finally, the metaplasticity of STDP was implemented in an NKN memristor with the priming spikes driven by the NKN NG. The NKN memristor with an NKN NG can be used as a sensor and a memory device in biomedical applications to detect various mechanical stimuli in the human body as well as to store data to provide feedback for medical treatments.

\section{CONFLICT OF INTEREST}

The authors declare no conflict of interest.

\section{ACKNOWLEDGEMENTS}

This research was supported by Basic Science Research Program through the National Research Foundation of Korea (NRF) funded by the Ministry of Science, ICT \& Future Planning (2017R1A2B4007189). We thank the KU-KIST graduate school program of Korea University.

1 Zheng, Q., Shi, B., Fan, F., Wang, X., Yan, L., Yuan, W., Wang, S., Liu, H., Li, Z. \& Wang, Z. L. In vivo powering of pacemaker by breathing-driven implanted triboelectric nanogenerator. Adv. Mater. 26, 5851-5856 (2014).

2 Hinchet, R. \& Kim, S.-W. Wearable and implantable mechanical energy harvesters for self-powered biomedical systems. ACS Nano 9, 7742-7745 (2015).

3 Hwang, G. T., Park, H., Lee, J. H., Oh, S., Park, K. I., Byun, M., Park, H., Ahn, G., Jeong, C. K., No, K., Kwon, H., Lee, S. G., Joung, B. \& Lee, K. J. Self-powered cardiac pacemaker enabled by flexible single crystalline PMN-PT piezoelectric energy harvester. Adv. Mater. 26, 4880-4887 (2014). 
4 Wang, Z. L. Towards self-powered nanosystems: from nanogenerators to nanopiezotronics. Adv. Funct. Mater. 18, 3553-3567 (2008).

5 Yang, R., Qin, Y., Li, C., Zhu, G. \& Wang, Z. L. Converting biomechanical energy into electricity by a muscle-movement-driven nanogenerator. Nano Lett. 9, 1201-1205 (2009).

6 Legenstein, R. Computer science: nanoscale connections for brain-like circuits. Nature 521, 37-38 (2015).

7 Backus, J. Can programming be liberated from the von Neumann style? A functional style and its algebra of programs. Commun. ACM 21, 613-641 (1978).

8 Hasegawa, T., Terabe, K., Tsuruoka, T. \& Aono, M. Atomic switch: atom/ion movement controlled devices for beyond Von-Neumann computers. Adv. Mater. 24, 252-267 (2012).

9 Mead, C. Neuromorphic electronic systems. Proc. IEEE 78, 1629-1636 (1990).

10 Prezioso, M., Merrikh-Bayat, F., Hoskins, B. D., Adam, G. C., Likharev, K. K. \& Strukov, D. B. Training and operation of an integrated neuromorphic network based on metal-oxide memristors. Nature 521, 61-64 (2015).

$11 \mathrm{Bi}$, G. \& Poo, M. J. Synaptic modifications in cultured hippocampal neurons: dependence on spike timing, synaptic strength, and postsynaptic cell type. Neuroscience 18, 10464-10472 (1998).

12 Abbott, L. F. \& Nelson, S. B. Synaptic plasticity: taming the beast. Nat. Neurosci. 3, 1178-1183 (2000)

13 Watt, A. J. \& Desai, N. S. Homeostatic plasticity and STDP: keeping a neuron's cool in a fluctuating world. Front. Synaptic Neurosci 2, 240-255 (2010).

14 Abraham, W. C. Metaplasticity: tuning synapses and networks for plasticity. Nat. Rev. Neurosci. 9, 387-387 (2008)

15 Abraham, W. C. \& Bear, M. F. Metaplasticity: the plasticity of synaptic plasticity. Trends Neurosci. 19, 126-130 (1996).

16 Abraham, W. C. \& Tate, W. P. Metaplasticity: a new vista across the field of synaptic plasticity. Prog. Neurobiol. 52, 303-323 (1997).

17 Jo, S. H., Chang, T., Ebong, I., Bhadviya, B. B., Mazumder, P. \& Lu, W. Nanoscale memristor device as synapse in neuromorphic systems. Nano Lett. 10 , 1297-1301 (2010).

18 Strukov, D. B., Snider, G. S., Stewart, D. R. \& Williams, R. S. The missing memristor found. Nature 453, 80-83 (2008).

19 Ohno, T., Hasegawa, T., Tsuruoka, T., Terabe, K., Gimzewski, J. K. \& Aono, M. Short-term plasticity and long-term potentiation mimicked in single inorganic synapses. Nat. Mater. 10, 591-595 (2011).

20 Kim, D. J., Lu, H., Ryu, S., Bark, C.-W., Eom, C.-B., Tsymbal, E. Y. \& Gruverman, A. Ferroelectric tunnel memristor. Nano Lett. 12, 5697-5702 (2012).

21 Yu, S., Gao, B., Fang, Z., Yu, H. Y., Kang, J. \& Wong, H. S. P. A low energy oxide-based electronic synaptic device for neuromorphic visual systems with tolerance to device variation. Adv. Mater. 25, 1774-1779 (2013).

22 Wang, Z. Q., Xu, H. Y., Li, X. H., Yu, H., Liu, Y. C. \& Zhu, X. J. Synaptic learning and memory functions achieved using oxygen ion migration/diffusion in an amorphous InGaZnO memristor. Adv.Funct. Mater. 22, 2759-2765 (2012).

23 Jeong, H. Y., Lee, J. Y. \& Choi, S.-Y. Interface-engineered amorphous $\mathrm{TiO}_{2}$-based resistive memory devices. Adv. Funct. Mater. 20, 3912-3917 (2010).

24 Miao, F., Strachan, J. P., Yang, J. J., Zhang, M., Goldfarb, I., Torrezan, A. C., Eschbach, P., Kelley, R. D., Medeiros-Ribeiro, G. \& Williams, R. S. Anatomy of a nanoscale conduction channel reveals the mechanism of a high-performance memristor. Adv. Mater. 23, 5633-5640 (2011).

25 Wright, C. D., Liu, Y., Kohary, K. I., Aziz, M. M. \& Hicken, R. J. Arithmetic and biologically-inspired computing using phase-change materials. Adv. Mater. 23, 3408-3413 (2011).
26 Indiveri, G., Chicca, E. \& Douglas, R. A VLSI array of low-power spiking neurons and bistable synapses with spike-timing dependent plasticity. IEEE Trans. Neural Netw. 17 211-221 (2006).

27 Tan, Z.-H., Yang, R., Terabe, K., Yin, X.-B., Zhang, X.-D. \& Guo, X. Synaptic metaplasticity realized in oxide memristive devices. Adv. Mater. 28, 377-384 (2016).

28 Kim, B. Y. Lee, W. H. Hwang, H. G. Kim, D. H. Kim, J. H. Lee, S. H. \& Nahm, S. Resistive switching memory integrated with nanogenerator for self-powered bioimplantable devices. Adv. Funct. Mater. 26, 5211-5221 (2016).

29 Li, X., Tao, J., Guo, W., Zhang, X., Luo, J., Chen, M., Zhu, J. \& Pan, C. A Self-powered system based on triboelectric nanogenerators and supercapacitors for metal corrosion prevention. J. Mater. Chem. A 3, 22663-22668 (2015).

30 Guo, W. X., Li, X. Y., Chen, M. X., Xu, L., Dong, L., Cao, X., Tang, W., Zhu, J., Lin, C. J., Pan, C. F. \& Wang, Z. L. Electrochemical cathodic protection powered by triboelectric nanogenerator. Adv. Funct. Mater. 24, 6691-6700 (2014).

31 Wang, Z., Joshi, S., Savel'ev, S. E., Jiang, H., Midya, R., Lin, P., Hu, M., Ge, N., Strachan, J. P., Li, Z., Wu, Q., Barnell, M., Li, G.-L., Xin, H. L., Williams, R. S., Xia, Q. \& Yang, J. J. Memristors with diffusive dynamics as synaptic emulators for neuromorphic computing. Nat. Mater. 16, 101-108 (2017).

32 Bliss, T. V. P. \& Collingridge, G. L. A synaptic model of memory: long-term potentiation in the hippocampus. Nature 361, 31-39 (1993).

33 Stevens, C. F. \& Wesseling, J. F. Augmentation is a potentiation of the exocytotic process. Neuron 22, 139-146 (1999).

34 Chang, T., Jo, S. H. \& Lu, W. Short-term memory to long-term memory transition in a nanoscale memristor. ACS Nano 5, 7669-7676 (2011).

35 Sjöström, P. J., Turrigiano, G. G. \& Nelson, S. B. Rate, timing, and cooperativity jointly determine cortical synaptic plasticity. Neuron 32, 1149-1164 (2001).

36 Caporale, N. \& Dan, Y. Spike timing-dependent plasticity: a Hebbian learning rule. Annu. Rev. Neurosci. 31, 25-46 (2008).

37 Xu, H. B., Ko, Y. J., Lee, T. G., Park, S. J., Noh, M. S., Kim, B. Y., Kim, J. S. \& Nahm, S. Structural and piezoelectric properties of $\left(\mathrm{Na}_{1-x} \mathrm{~K}_{\mathrm{x}}\right) \mathrm{NbO}_{3}$ platelets and their application for piezoelectric nanogenerator. J. Am. Ceram. Soc. 99, 3476-3484 (2016).

38 Jeon, H., Park, J., Jang, W., Kim, H., Kang, C., Song, H., Kim, H., Seo, H. \& Jeon, H. Stabilized resistive switching behaviors of a Pt/TaOx/TiN RRAM under different oxygen contents. Phys. Stat. Sol 211, 2189-2194 (2014).

39 Bousoulas, P., Michelakaki, I. \& Tsoukalas, D. Influence of oxygen content of room temperature $\mathrm{TiO}_{2-x}$ deposited films for enhanced resistive switching memory performance. J. Appl. Phys. 115, 034516 (2014).

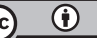

This work is licensed under a Creative Commons Attribution 4.0 International License. The images or other third party material in this article are included in the article's Creative Commons license, unless indicated otherwise in the credit line; if the material is not included under the Creative Commons license, users will need to obtain permission from the license holder to reproduce the material. To view a copy of this license, visit http:// creativecommons.org/licenses/by/4.0/

(C) The Author(s) 2017

Supplementary Information accompanies the paper on the NPG Asia Materials website (http://www.nature.com/am) 